\title{
Bi-functional Activities of Chimeric Lysozymes Constructed by Domain Swapping between Bacteriophage T7 and K11 Lysozymes
}

\author{
Ethel H. Alcantara ${ }^{1}$, Dong Hee Kim ${ }^{1}$, Su-Il Do $^{2}$ and Sang Soo Lee ${ }^{1, *}$ \\ ${ }^{1}$ Research Center for Bio-Medicinal Resources and Department of Life Science and Technology, Pai Chai University, \\ 439-6 Doma-dong, Seo-gu, Daejeon 302-735, Korea \\ ${ }^{2}$ Department of Life science, Laboratory of functional Glycomics, Ajou Unversity, \\ San 5 Wonchun-dong, Youngtong-gu, Suwon 443-749, Korea
}

Received 22 January 2006, Accepted 23 February 2007

The lysozymes encoded by bacteriophage T7 and K11 are both bifunctional enzymes sharing an extensive sequence homology $(\mathbf{7 5} \%)$. The constructions of chimeric lysozymes were carried out by swapping the $\mathrm{N}$-terminal and $\mathrm{C}$ terminal domains between phage $\mathrm{T} 7$ and K11 lysozymes. This technique generated two chimeras, T7K11-lysozyme (N-terminal T7 domain and C-terminal K11 domain) and K11T7-lysozyme (N-terminal K11 domain and C-terminal T7 domain), which are both enzymatically active. The amidase activity of T7K11-lysozyme is comparable with the parental enzymes while K11T7-lysozyme exhibits an activity that is approximately $45 \%$ greater than the wildtype lysozymes. Moreover, these chimeric constructs have optimum pH of 7.2-7.4 similar to the parental lysozymes but exhibit greater thermal stabilities. On the other hand, the chimeras inhibit transcription comparable with the parental lysozymes depending on the source of their $\mathrm{N}$ terminals. Taken together, our results indicated that domain swapping technique localizes the $\mathrm{N}$-terminal region as the domain responsible for the transcription inhibition specificity of the wild type T7 and K11 lysozymes. Furthermore, we were able to develop a simple and rapid purification scheme in purifying both the wild-type and chimeric lysozymes.

Keywords: Amidase, His-tagging, K11 RNA polymerase, Phage K11 lysozyme, Transcription inhibition

In this paper N-terminal histidine tagged $\mathrm{K} 11, \mathrm{~T} 7$ and chimeric lysozymes by swapping the $\mathrm{N}$-terminal and $\mathrm{C}$-terminal domains between T7 and K11 lysozymes were constructed. These lysozymes were purified and characterized.

*To whom correspondence should be addressed.

Tel: 82-42-520-5616; Fax: 82-42-520-5666

E-mail: sslee@pcu.ac.kr

\section{Introduction}

The bacteriophage group that includes $\mathrm{T} 7$ and K11 produces two important proteins that have been widely used as models for the investigation of the physicochemical aspect of transcription. These proteins are the DNA-dependent RNAP (RNA polymerase) (Chamberlin et al., 1970; Dietz et al. 1990) and lysozyme (Inouye et al., 1973; Junn et al., 2005). The bacteriophage RNAPs are single-subunit proteins that are able to perform the complete transcriptional cycle in the absence of additional protein factors and show stringent specificity toward their promoters (Rosa and Andrews, 1981; Dunn and Studier, 1983; Brown et al., 1986; Han et al., 2002). On the other hand, the lysozymes encoded by bacteriophage T7 and K11 are both bifunctional enzymes. They possess amidase activity and bind specifically to their respective RNAPs and inhibit transcription (Ikeda and Bailey, 1992; Cheng et al., 1993; Huang et al., 1999; Junn et al., 2005) and T7 lysozyme stimulates replication and packaging of T7 DNA (Zhang and Studier, 2004).

The bacteriophage T7 lysozyme has already been extensively studied. Its X-ray crystallographic data revealed that $\mathrm{T} 7$ lysozyme contains a zinc atom necessary for amidase activity but not for inhibition of T7 RNAP (Junn et al., 2005). The crystal structure of T7 lysozyme-T7 RNAP complex has also been elucidated revealing that lysozyme binds at a site remote from the polymerase active site suggesting an indirect mechanism of inhibition (Jeruzalmi and Steitz, 1998; Junn et al., 2005). Also, mutational analysis identified that the $\mathrm{N}$ terminus of T7 lysozyme gene appeared to be important in interacting with T7 RNAP (Cheng et al., 1994). T7 RNAP mutants that have an increased or decreased sensitivity to lysozyme inhibition have been isolated during T7 phage infection (Moffatt and Studier, 1987; Zhang and Studier, 1995; Zhang and Studier, 2004). The complex between T7 lysozyme and T7 RNAP is essential and tightly regulated in 
phage growth (Moffatt and Studier, 1987). Thus, mutant T7 RNAP or lysozyme unable to form the complex were isolated either by direct engineering or as suppressors (increased phage production) of hypersensitive polymerase mutants or over production of lysozyme (Zhang and Studier, 1995).

However, structural and functional studies on K11 lysozyme are relatively limited. The lysozyme gene of bacteriophage $\mathrm{K} 11$ was recently sequenced in our lab and the deduced amino acid sequence of its polypeptide shows $75 \%$ homology with the T7 lysozyme (Junn et al., 2005). The N-terminus of T7 lysozyme and the amino acid residues 30 to 42 which are found to be the site of interaction with T7 RNAP are conserved in K11 lysozyme. Moreover, Lys ${ }^{128}$ and $\mathrm{Tyr}^{46}$, which are important for amidase activity, are also conserved in K11 lysozyme. The highly conserved amino acid residues in the N-terminal and essential sites of T7 and K11 lysozyme genes suggest that both lysozymes have the same functional role in the bacteriophages (Junn et al., 2005).

In this study, we constructed a phage K11 lysozyme fused to a hexahistidine tag to facilitate single-step purification directly from the crude cell lysates. This method aimed to provide sufficient amount of protein for routine biochemical analysis. Although this technique is extremely useful for the rapid purification of lysozyme, the purification tag inevitably alters the primary sequence of the protein since two amino acid residues remain even after the tag is removed. We have therefore examined the effect of this tag on the amidase and RNAP inhibition activity of the enzyme. Moreover, since the two enzymes share a relatively high homology (75\%) and contain conserved essential sites (Junn et al., 2005), we also constructed chimeric enzymes by swapping the N-terminal and C-terminal domains between T7 and K11 lysozymes. The characterization of such chimeras would provide further insight into the effect of swapping and the role of each domain on amidase activity and RNAP inhibition activity of the lysozymes.

\section{Materials and Methods}

Construction of hexahistidine-tagged wild-type $\mathrm{K} 11$ and $\mathrm{T} 7$ lysozymes. Using pET-tacKLys (Junn et al., 2005) and pLyse (purchased from Novagen) plasmids as templates, $450 \mathrm{bp}$ fragments containing the $\mathrm{K} 11$ and $\mathrm{T} 7$ lysozyme genes were amplified by polymerase chain reaction using Taq-EF DNA polymerase (purchased from Takara) respectively. The sequences of the forward and reverse primers for K11 lysozyme gene are 5'GGAATTCCATATGGCCAAGGTTCAATTCA-3' and 5'-GCGGA TCCCTAATGTTTACCCACGGT-3' and those for T7 lysozyme gene are 5'-GGCGCATATGGCTCGTGTACAGTTT-3' and 5'-GCG GATCCTTATCCACGGTCAGAAGT-3', respectively. They were designed to anneal to upstream and downstream flanking sequences just outside the lysozyme gene and to contain NdeI and BamHI recognition sequences (underlined). The resulting PCR fragments were digested with $N d e I$ and $B a m H I$ restriction enzymes and were

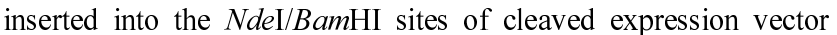

(A)

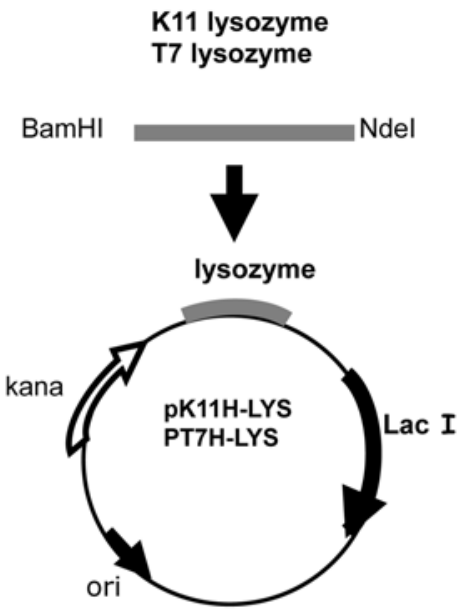

(B)

1

$56(\mathrm{Kpnl})$

151

lysozyme

$\Gamma 7$

K11

T7k11

K11T7

Fig. 1. (A) Structure of expression vectors for the N-terminal histidine tagged K11 and T7 lysozyme genes. The pK11H-LYS and pT7H-LYS plasmids contain the hexahistidine tagged K11 and T7 lysozyme gene, respectively, under the control of lac promoter. They also carry the bla gene for ampicillin resistance and the lacI gene for the lac repressor. (B) Construction of chimeric lysozymes of swapping the N-terminal and C-terminal domains between $\mathrm{T} 7$ and K11 lysozymes.

pET28a (purchased from Novagen) and the resulting recombinant plasmids were named pK11H-LYS for K11 lysozyme and pT7HLYS for T7 lysozyme (Fig. 1A).

Construction of domain swapping mutants. To swap the Nterminal and C-terminal domains between $\mathrm{T} 7$ and $\mathrm{K} 11$ lysozymes, a $K p n I$ restriction site was introduced in $\mathrm{T} 7$ lysozyme by sitedirected mutagenesis using overlap extension PCR method. Two outside primers are same primers used for the construction of pT7H-LYS and each contains NdeI and BamHI restriction site respectively. Two internal primers with mismatched base pair sequence (in bold), forward primer 5'- CAAGCGAGACGGTACC GTGGAGGCAG-3' and reverse primer 5'-CCTCCACGGTACCG TCTCGCTTGATG-3', were designed to introduce a single base substitution thus generating a KpnI restriction site (GGTACC) at the position where the same restriction site is present in the nucleotide sequence of K11 lysozyme. DNA sequencing confirmed that the necessary substitution was incorporated in the T7 lysozyme gene.

To construct the chimeric lysozymes, the pK11H-LYS vector containing the wild type K11 lysozyme and the PCR-generated mutant T7 lysozyme were both digested with $\mathrm{KpnI}$ and $\mathrm{NdeI}$ 
restriction enzymes. The T7 lysozyme fragment (168 bp) containing the N-terminal domain of the enzyme was then inserted and cloned into the cleaved pK11H-LYS generating the T7K11-lysozyme mutant. This chimeric enzyme contains 56 amino acids of T7 lysozyme N-terminal and 95 amino acids of K11 lysozyme Cterminal (Fig. 1B). Accordingly, to generate the other domain swapping mutant, the pK11H-LYS vector and the mutant T7 lysozyme was cleaved with $K p n \mathrm{I}$ and BamHI restriction enzymes. The T7 lysozyme fragment ( $285 \mathrm{bp})$ generated in this restriction digestion was cloned into the previously digested pK11H-LYS generating the K11T7-lysozyme mutant. This domain swapped enzyme contained 56 amino acids of K11 lysozyme N-terminal and 95 amino acids of K11 lysozyme C-terminal (Fig. 1B).

Expression and purification of hexahistidine-tagged K11 and chimeric lysozymes. E. coli BL21 (DE3) cells were transformed respectively with pK11H-LYS, pT7K11H-LYS, and pK11T7H-LYS and grown on LB plates containing kanamycin $(50 \mu \mathrm{g} / \mathrm{ml})$. Overnight cultures in $5 \mathrm{ml}$ LB broth were started with single colonies and used to inoculate expression cultures $(200 \mathrm{ml})$. When the culture reached $\mathrm{A}_{600}=0.5$, IPTG was added to a final concentration of $0.1 \mathrm{mM}$ and cultivation was allowed to proceed for $4 \mathrm{~h}$ at $37^{\circ} \mathrm{C}$.

After induction, cells were harvested by centrifugation and resuspended in buffer A containing $20 \mathrm{mM}$ Tris- $\mathrm{HCl}$, pH 8.0, $1 \mathrm{mM}$ EDTA, $1 \mathrm{mM}$ DTT, and 5\% glycerol. The cells were then sonicated on ice using a microtip with $50 \%$ power for five 30 -s interval and soluble and insoluble fractions were separated by centrifugation. The pellet fraction was stored at $-80^{\circ} \mathrm{C}$ until use. Whole cell lysates, induced and uninduced, and soluble and insoluble fractions were analyzed by $15 \%$ SDS-PAGE and bands were visualized by Coomassie blue staining.

The hexahistidine-tagged K11 and chimeric lysozyme proteins were purified under denaturating conditions using Ni-NTA affinity chromatography. All steps were carried out at $0-4^{\circ} \mathrm{C}$. Frozen pellet cells were thawed on ice and resuspended in $50 \mathrm{ml}$ of buffer containing $25 \mathrm{mM}$ Tris-HCl, pH 8.0, $100 \mathrm{mM} \mathrm{NaCl}, 2 \mathrm{mM}$ EDTA, and $7 \mathrm{mM} \beta$-mercaptoethanol, and repelleted by centrifugation $(15,000 \mathrm{rpm}$ for $30 \mathrm{~min})$. After washing twice using this procedure, the pellet was solubilized with $20 \mathrm{ml}$ of Solution A containing $8 \mathrm{M}$ urea, $10 \mathrm{mM}$ Tris-Cl, $\mathrm{pH} 8.0$, and $100 \mathrm{mM}$ Na-phosphate and was incubated on ice with gentle shaking for $1 \mathrm{~h}$. Insoluble materials were removed by centrifugation (15,000 rpm for $30 \mathrm{~min}$ ).

Three milliliters of Ni-NTA agarose (purchased from Qiagen) was added to the clarified supernatant and the mixture was gently shaken on ice for $1 \mathrm{~h}$ to facilitate binding. The mixture was then loaded on an empty column and the unbound protein was eluted. Nonspecific proteins that bound with the Ni-NTA agarose were eluted by washing the column with $100 \mathrm{ml}$ of Solution A at pH 6.3. The hexahistidine-tagged K11 and chimeric lysozymes were finally eluted with $10 \mathrm{ml}$ of Solution $\mathrm{A}$ at $\mathrm{pH} 4.5$. The fractions were examined by $15 \%$ SDS-PAGE and the appropriate fractions were pooled and refolded by dialyzing against a buffer containing 25 $\mathrm{mM}$ Tris- $\mathrm{HCl}, \mathrm{pH}$ 8.0, $2 \mathrm{mM}$ EDTA, $2 \mathrm{M}$ urea, $7 \mathrm{mM} \beta-$ mercaptoethanol, and $20 \%$ glycerol. After a 3 -h dialysis using this solution, the proteins were transferred and dialyzed overnight against a buffer containing $50 \mathrm{mM}$ Tris- $\mathrm{HCl}, \mathrm{pH}$ 8.0, 2 mM EDTA, $7 \mathrm{mM} \beta$-mercaptoethanol, and $20 \%$ glycerol. The $\mathrm{N}$-terminal tag of the lysozymes was cleaved by adding $20 \mu \mathrm{l}$ of thrombin (purchased from Sigma) and incubating at $4^{\circ} \mathrm{C}$ for $22 \mathrm{~h}$. The cleaved tag and thrombin were removed by passing the reaction mixture in $200 \mu \mathrm{l}$ of DEAE resin. The purified thrombin cleaved protein was stored in aliquots at $-20^{\circ} \mathrm{C}$ until use for amidase and in vitro transcription assays.

Expression and purification of hexahistidine-tagged wild-type T7 lysozyme. E. coli BL21 (DE3) cells were transformed with pT7H-LYS containing HIS-tagged T7 lysozyme gene and grown on LB plates containing kanamycin $(50 \mu \mathrm{g} / \mathrm{ml})$. The cultivation and induction procedures of K11 lysozyme gene described above were also used for the cultivation and expression of T7 lysozyme gene.

The histidine tagged T7 lysozyme was mostly found in the soluble supernatant fraction thus it was purified under non-denaturating conditions using Ni-NTA affinity chromatography. The soluble fraction was mixed with $3 \mathrm{ml}$ of Ni-NTA agarose that had been previously washed and equilibrated with buffer containing $50 \mathrm{mM}$ Tris- $\mathrm{Cl}$ (pH 8), $300 \mathrm{mM} \mathrm{NaCl}$, and $20 \mathrm{mM}$ imidazole. The reaction mixture was gently shaken on ice for $1 \mathrm{~h}$ to facilitate binding. The mixture was then poured into an empty column and the unbound fraction was eluted. The resin was then washed with $100 \mathrm{ml}$ of the previous buffer to remove nonspecific binding. The $\mathrm{T} 7$ lysozyme was finally eluted with elution buffer containing $50 \mathrm{mM}$ Tris-Cl (pH 8), $300 \mathrm{mM} \mathrm{NaCl}$ and $250 \mathrm{mM}$ imidazole. The appropriate protein fractions were then pooled and dialyzed against a buffer containing $50 \mathrm{mM}$ Tris-Cl, $100 \mathrm{mM} \mathrm{NaCl}, 0.1 \%$ Triton-X, $7 \mathrm{mM}$ $\beta$-mercaptoethanol, and 50\% glycerol. The purified T7 lysozyme was cleaved with thrombin and was passed through a DEAE resin. The purified thrombin cleaved protein was stored in aliquots at $-20^{\circ} \mathrm{C}$ until use for amidase and in vitro transcription assays.

Amidase assay. The amidase activity was assayed by measuring the decrease in turbidity of E. coli XL-1 blue cells that were incubated in $0.1 \mathrm{M}$ EDTA, pH 8.0 for $5 \mathrm{~min}$ at room temperature, pelleted, and resuspended in PBS buffer and kept in ice until use (Cheng et al., 1993; Junn et al., 2005). To measure cell wall lysis activity, various concentrations of phage lysozymes, were added to $1 \mathrm{ml}$ of sensitized cells that had been brought to room temperature and the decrease in $\mathrm{OD}_{600}$ was monitored. For amidase assay at elevated temperatures, the enzyme was incubated for $10 \mathrm{~min}$ at various temperatures. One unit of activity was defined as the amount of enzyme that reduced $\mathrm{OD}_{600}$ at the rate of $0.001 / \mathrm{min}$.

In vitro transcription assay. A $20 \mu \mathrm{l}$ standard transcription reaction containing $40 \mathrm{mM}$ Tris- $\mathrm{Cl} \mathrm{pH} 8,8 \mathrm{mM} \mathrm{MgCl} 2,20 \mathrm{mM}$ spermidine, $10 \mathrm{mM}$ DTT, $0.5 \mathrm{mM}$ NTP, $2 \mu \mathrm{Ci}$ of $\left[\alpha-{ }^{32} \mathrm{P}\right] \mathrm{CTP}(30$ $\mu \mathrm{C} / \mu \mathrm{M}), 0.1 \mu \mathrm{g}$ of template DNA, $10 \mathrm{U}$ of T7 or K11 RNAP purified using the method previously described (Han et al., 1999), and variable amounts of lysozyme was used. The reactions were initiated by adding the RNAP and the reaction mixtures were incubated at $37^{\circ} \mathrm{C}$ for $30 \mathrm{~min}$. The reaction were terminated by adding 10\% TCA solution and the mixtures were incubated on ice for $10 \mathrm{~min}$. The solution was then filtered and the amount of RNA synthesized was determined by measuring the amount of radioactivity retained on the GF/B filter (purchased from Whatman) by a Beckman multi-purpose Scintillation counter LS 6500 . 


\section{Results}

Cloning and expression of hexahistidine-tagged K11, T7 and chimeric lysozymes. The bacteriophage K11 lysozyme gene was amplified by PCR using pK11H-LYS as template. The amplified lysozyme gene, which was engineered to contain NdeI and BamHI recognition sequences, was cloned into a pET-28a vector under the control of T7 promoter.

The wild type K11 lysozyme has a KpnI (GGTACC) recognition sequence at bp 164-169 which encodes the amino acids $\mathrm{Gly}^{55}$ and $\mathrm{Thr}^{56}$. These amino acid residues are conserved in $\mathrm{T} 7$ lysozyme but $\mathrm{Thr}^{56}$ is encoded by a different codon thus the corresponding nucleotide sequence for $\mathrm{T} 7$ lysozyme is GGTACT. To introduce a $K p n \mathrm{I}$ site in T7 lysozyme, we employed site-directed mutagenesis by overlap PCR to generate a single base substitution (GGTACT to GGTACC). This procedure introduced one silent point mutation in the coding region of T7 lysozyme changing ACT triplets encoding $\mathrm{Thr}^{56}$ to the synonymous codon ACC. The lysozyme genes were then cut in this region by KpnI restriction enzyme digestion and their respective domains were swapped (Fig. 1B). Two chimeric enzymes were generated, one which contains a T7 N-terminal and a K11 Cterminal and another which contains a K11 N-terminal and T7 C-terminal and were named T7K11-lysozyme and K11T7lysozyme, respectively. Cloning of each chimera was confirmed by cutting with restriction enzyme unique to each swapped domain; AflII for K11T7-lysozyme and DraI for T7K11lysozyme.

E. coli BL21 (DE3) cells harboring pK11H-LYS were cultured at $37^{\circ} \mathrm{C}$ in the presence and absence of IPTG, and the whole cell lysates were analyzed by $15 \%$ SDS-PAGE as shown in Fig. 2A. One major band appeared at approximately $21 \mathrm{kDa}$, a size slightly higher than the actual protein size since the lysozyme is tagged and carries 15 additional amino acid residues on its N-terminal. Although the lysozyme was efficiently expressed at $37^{\circ} \mathrm{C}$, almost all of the lysozyme fractions aggregated as inclusion bodies showing the same results of previously reported in our lab (Junn et al., 2005). On the other hands, $\mathrm{T} 7$ lysozyme is induced at $37^{\circ} \mathrm{C}$ and mostly found in the soluble supernatant fractions (Fig. 2A). Also, both of the chimeric lysozymes are induced at $37^{\circ} \mathrm{C}$ and most lysozyme fractions are aggregated as inclusion bodies (Fig 2A).

Inclusion body formation is a common problem encountered in expressing recombinant proteins in E. coli. Several alterations of the expression protocol were tried in increasing the amount of soluble protein. Decreasing the induction temperature did not produce a substantial amount of soluble lysozyme (data not shown).

A possible strategy for the prevention of protein aggregation is the co-expression of molecular chaperones. We co-transformed the plasmid containing the lysozyme gene with pGroESL, a plasmid containing the GroES and GroEL genes which are known to enhance protein solubility. However, this technique
(A)
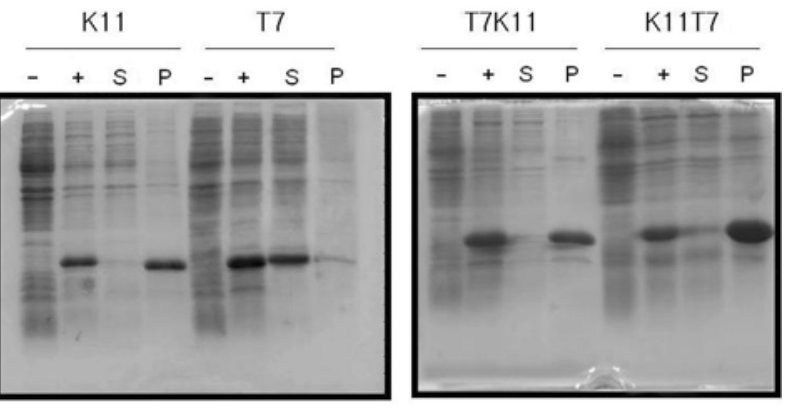

(B)
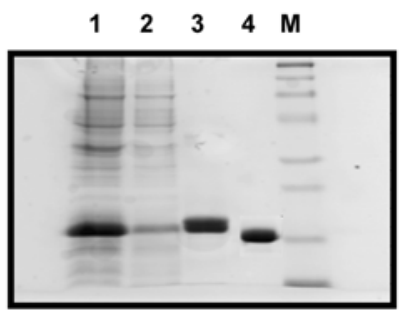

Fig. 2. (A) SDS-PAGE analysis of $\mathrm{K} 11, \mathrm{~T} 7$ and chimeric lysozymes. K11, T7, T7K11, K11T7 lysozymes are induced at $37^{\circ} \mathrm{C}$. Their genes were either induced $(+)$ or uninduced (-) with IPTG; soluble (S) and insoluble (P) fractions were separated by centrifugation. Equal amounts of protein were loaded on each lane. (B) Purification of hexahistidine-tagged K11 lysozyme. The inclusion bodies were solubilized in $8 \mathrm{M}$ urea and purified in NiNTA column under denaturating condition. Lane 1, inclusion bodies solubilized with $8 \mathrm{M}$ urea: lane 2, Ni-NTA unbound fraction: lane 3, Ni-NTA elution fraction; lane 4, cleaved lysozyme; lane $\mathrm{M}$, protein size marker

again failed to prevent aggregation and to promote proper folding since most of the lysozyme remained in the pellet fraction (data not shown). The insolubility of K11 lysozyme in E. coli might have been caused by its difference in amino acid sequence and in its natural host (Klebsiella for phage K11) (Junn et al., 2005). Moreover, it seemed to be likely that K11 lysozyme belongs to a group of proteins that fold improperly upon intracellular expression in $E$. coli.

Purification of hexahistidine-tagged K11, T7 and chimeric lysozymes. Since various techniques in producing soluble lysozyme did not give favorable results, the protein was purified from inclusion bodies. The pellet was solubilized in 8 $\mathrm{M}$ urea and was fractionated by Ni-affinity chromatography under denaturating condition. The purification of K11 lysozyme is summarized in Table 1 . Approximately $13.5 \mathrm{mg}$ of homogenous K11 lysozyme was obtained from a 200-ml cell culture. Fractions of eluted protein were analyzed by $15 \%$ SDS-PAGE and stained with Coomassie blue. As shown in Fig. 2 B, approximately $97 \%$ homogenous K11 lysozyme was recovered in a single-step elution. The elution fractions resulted in a single protein band. Thus, with the use of hexahistidine tag, we were able to develop a simple and rapid purification for K11 lysozyme to be used for further 
Table 1. Purification of tagged bacteriophage K11 lysozyme

\begin{tabular}{lcccc}
\hline \multicolumn{1}{c}{ Step } & $\begin{array}{c}\text { Total } \\
\text { protein } \\
(\mathrm{mg})\end{array}$ & $\begin{array}{c}\text { Total } \\
\text { activity } \\
\text { (units) }\end{array}$ & $\begin{array}{c}\text { Specific } \\
\text { activity } \\
\text { (units/mg) }\end{array}$ & $\begin{array}{c}\text { Protein } \\
\text { yield } \\
(\%)\end{array}$ \\
\hline Crude extract & 620 & - & - & 100 \\
Inclusion body & 152 & - & - & 24.5 \\
Ni-NTA elute & 13.5 & 19,400 & 1,437 & 2.1 \\
\hline
\end{tabular}

biochemical characterization of the enzyme.

Refolding of the denatured lysozyme was carried out by dialysis with a buffer containing an intermediate denaturant concentration (Clark, 1998). Previous refolding of K11 lysozyme resulted in aggregation (Junn et al., 2005); however, with the presence of $2 \mathrm{M}$ urea in the dialysis buffer, we were able to refold the lysozyme without any observed aggregation. This implied that the denaturant concentration was high enough to solubilize aggregates but low enough to promote proper folding. The protein solution was then transferred to the same buffer without the presence of the denaturant and dialysis was continued until the protein refolded completely.

The relative specific activity of the purified lysozyme was estimated to be $1437 \mathrm{U} / \mathrm{mg}$ (Table 1). Compared to the previously purified wild-type lysozyme in our laboratory; this activity is about $60 \%$ higher. Previously purified K11 lysozyme without hexahistidine was reported to have a specific activity of $900 \mathrm{U} / \mathrm{mg}$ (Junn et al., 2005). The observed increase in enzyme activity might be attributed in the presence of the tag. Affinity tags can be helpful for stabilizing proteins or enhancing their solubility (Terpe, 2003). Even upon cleaving the hexahistidine tag, the N-terminal of the lysozyme still has two extra amino acid residues (Gly-Ser) which, based on our results, can still exert the same effect.

Both chimeras were efficiently purified by using the same purification method of K11 lysozyme but the protein yield of K11T7-lysozyme was lower compared with T7K11-lysozyme and the parental lysozymes. A 200-ml cell culture yielded $13.1 \mathrm{mg}$ T7K11-lysozyme while similar amount of culture yielded $9.3 \mathrm{mg}$ K11T7-lysozyme. The protein yield of wild type T7 and K11 lysozymes is $16.5 \mathrm{mg}$ and $13.5 \mathrm{mg}$, respectively (data not shown). Differences in purification profiles may be due to modifications of the enzyme conformations caused by the interaction of domains derived from the different wild type proteins (Berger et al., 2001). The homology between T7 and K11 lysozymes corresponding with the swapped domain in Nterminal part (1-56 amino acids) is $78 \%$ while in C-terminal part (57-151 amino acids) is only $66 \%$.

Amidase activities of wild type and chimeric lysozymes. To determine whether the purification tag affects the enzymatic function of the protein, purified lysozyme fractions with tag and with cleaved tag were assayed as described in Materials and Methods. The amidase activity of tagged lysozyme and cleaved lysozyme were almost identical implying that the

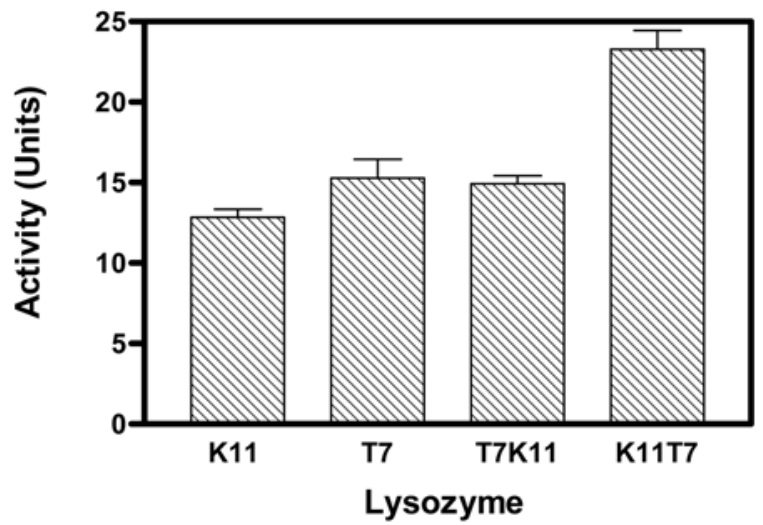

Fig. 3. Amidase activities of K11, T7 and chimeric lysozymes. The amidase activity was assayed by measuring the decrease in turbidity of $E$. coli XL-1 cell.

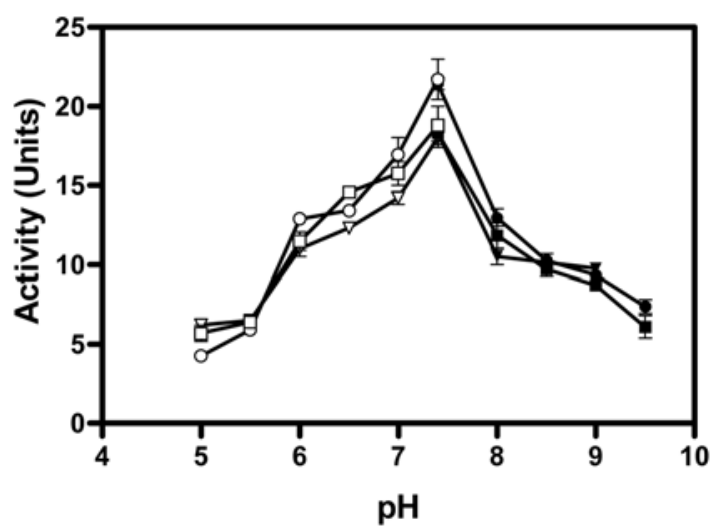

Fig. 4. $\mathrm{pH}$ profile of cell wall lysis activity of K11 and chimeric lysozymes. Closed symbol shows the activities measured in Tris$\mathrm{Cl}$ buffer ( $\mathrm{pH}$ 7.2-9.0) and open symbol shows the activities measured in PBS buffer (5.0-7.4). The amidase activities of K11 ( $\nabla$ and $\boldsymbol{\nabla}$ ), T7K11 hybrid ( $\square$ and $\boldsymbol{\square}$ ) and K11T7 ( $\bigcirc$ and hybrid were shown.

purification tag did not interfere with the enzyme function (data not shown). This result also suggests that the refolding process restored the activity of the protein.

The amidase activity of the chimeric lysozymes was assayed as previously described for wild type lysozymes (Cheng et al., 1993). As shown in Fig. 3, both chimeras possess amidase activities. The activity of T7K11-lysozyme was comparable with the activities of wild type T7 and K11 lysozymes. On the other hand, the K11T7-lysozyme chimera exhibited a much higher activity that is approximately $45 \%$ greater than the parental lysozymes.

We also examined the effect of $\mathrm{pH}$ on the enzymatic activity of the protein. Sensitized $E$. coli XL-1 blue cells were resuspended in different buffers at various $\mathrm{pH}$; $\mathrm{PBS}$ buffer at $\mathrm{pH}$ 5.0-7.4; and Tris-Cl buffer at $\mathrm{pH}$ 7.2-9.0. The optimal $\mathrm{pH}$ for the enzyme activity of the purified wild type lysozyme was observed at $\mathrm{pH} 7.2$ to 7.4 (Fig. 4). The optimum $\mathrm{pH}$ 


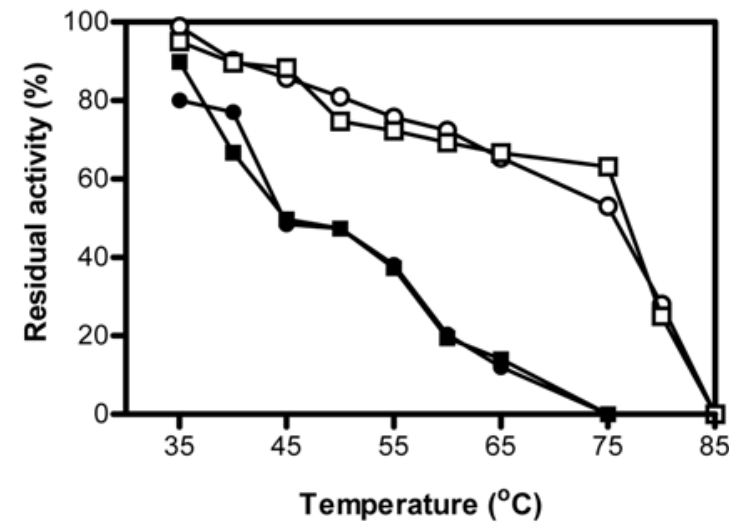

Fig. 5. Thermal stability of wild and chimeric lysozymes at various temperature. After incubation for $10 \mathrm{~min}$ at each temperature, cell wall lysis activities of $\mathrm{K} 11(\boldsymbol{O})$, T7 (ם), T7K11 ( $\square$ ) and K11T7 (○) lysozymes were measured.

observed for both chimeric lysozymes is similar to that of the parental lysozymes which is 7.2-7.4 (Fig. 4). Bacterial cell wall degradation at acidic or basic $\mathrm{pH}$ was also observed but the activity of the enzyme at these conditions was minimal.

The stability of the enzyme at elevated temperatures is shown on Fig. 5. The lysozyme fraction was incubated for 10 min at each indicated temperature and amidase activity was monitored. The amidase activity of the heat-treated lysozyme with hexahistidine tag was found to be more stable than the lysozyme with cleaved tag (data not shown). However, the chimeric lysozymes exhibited thermal stabilities significantly greater than the parental enzymes (Fig. 5). At $50^{\circ} \mathrm{C}$, the amidase activities of both wild type K11 and T7 lysozymes are decreased to $47 \%$ and $48 \%$, respectively. However, with the same temperature, the T7K11-lysozyme retained 75\% activity while the K11T7-lysozyme retained $81 \%$ activity. The parental lysozymes are both completely inactivated at $75^{\circ} \mathrm{C}$ while the chimeric lysozymes lost their amidase activities at $85^{\circ} \mathrm{C}$.

Transcription inhibition activities of wild type and chimeric lysozymes. The activities of T7 and K11 RNAPs were determined by measuring the conversion of radiolabeled NTPs into high molecular weight RNA that can be retained on GF/B Whatman filters (Ikeda and Bailey, 1992). Using this filter binding assay, the transcription of T7 and K11 DNA by their respective polymerase was monitored in the presence of increasing amounts of wild type and chimeric lysozymes.

Results from previous experiments revealed that phage $\mathrm{T} 7$ and K11 transcription systems are crossly regulated by their own lysozymes (Junn et al., 2005). That is, a certain extent, transcription by T7 RNAP could be regulated by K11 lysozyme and transcription by K11 RNAP could be regulated by T7 lysozyme. However, results also showed that these inhibitions are less compared with their respective lysozymes implying that inhibition is specific (Ikeda and Bailey, 1992; Junn et al., 2005).

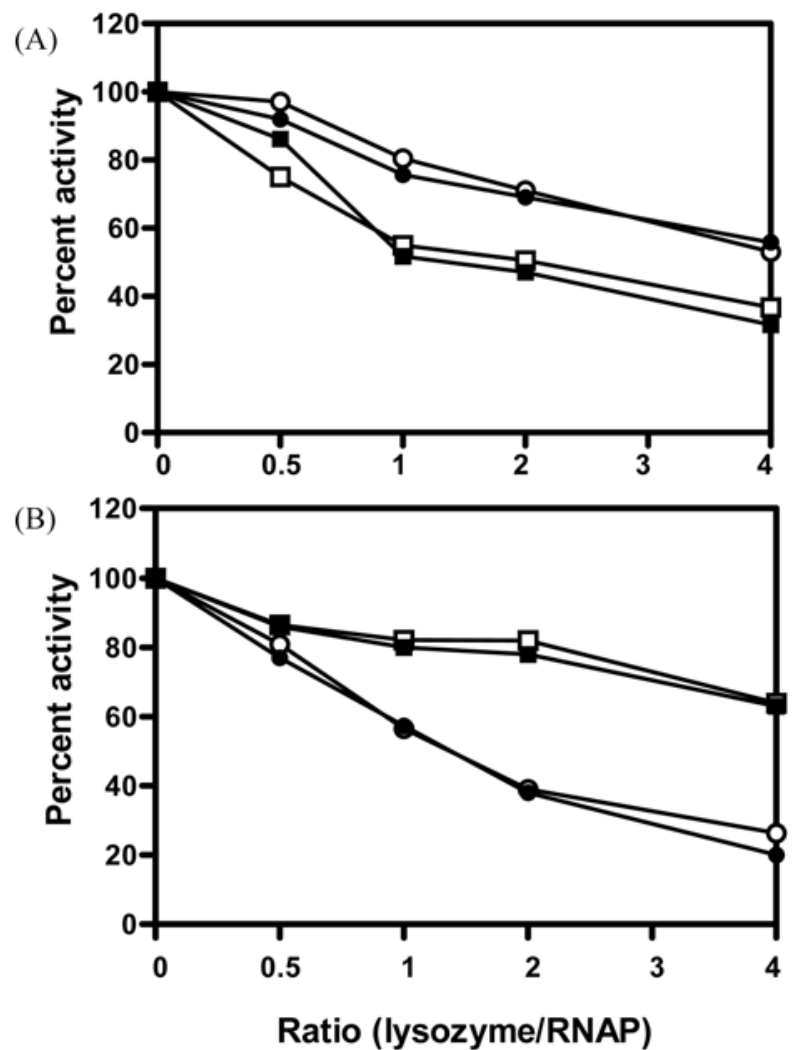

Fig. 6. (A) Inhibition of T7 RNA polymerase transcription by wild-type and chimeric lysozymes. One hundred percent activity for the T7 RNA polymerase is defined as the transcriptional activity measured in the absence of lysozyme. In the increasing ratio of K11 ( $)$, T7 (ם), T7K11 ( $\square$ ) and K11T7 (O) lysozymes to T7 RNAP, the relative transcription activities were measured respectively. (B) Inhibition of K11 RNAP transcription by wild-type and chimeric lysozymes. One hundred percent activity for the K11 RNAP is defined as the transcriptional activity measured in the absence of lysozyme. In the increasing ratio of K11 (O), T7 ( $\mathbf{\square})$, T7K11 ( $\square$ ) and K11T7 (O) lysozymes to K11 RNAP, the relative transcription activities were measured respectively.

The transcription inhibition activities of the wild type and chimeric lysozymes are shown on Fig. 6. With transcription by T7 RNAP, the T7K11-lysozyme exhibited inhibitory effect that is almost identical with the wild-type T7 lysozyme. At 1fold excess of T7 lysozyme and T7K11 lysozyme, transcription with T7 RNAP is decreased to $52 \%$ and $55 \%$, respectively. Similarly, with transcription by K11 RNAP, the K11T7lysozyme showed transcription inhibition that is comparable with wild type K11 lysozyme. At equimolar concentrations of K11 RNAP and K11 lysozyme, transcription activity is decreased to $57 \%$ while a 1 -fold excess of K11T7-lysozyme decreased transcription to $56 \%$.

Studier et al. reported earlier that the N-terminus of $\mathrm{T} 7$ lysozyme seems to be likely the site of interaction with T7 RNAP (Cheng et al., 1993). Since the T7K11-lysozyme 
contained the N-terminus of T7 lysozyme, it could be expected that it would exhibit inhibitory effect similar to the wild-type T7 lysozyme. The comparable inhibitory activities of K11 lysozyme and K11T7-lysozyme suggest that like T7 lysozyme, the N-terminus of K11 lysozyme could also be the site of interaction with K11 RNAP. However, it would be very interesting to note that the N-terminal of both wild type lysozymes share a high degree of homology (80\%) but are nonetheless specific in inhibiting transcription by their respective RNAPs.

\section{Discussion}

Routine biochemical characterization of enzymes often requires rapid and simple purification scheme. Isolation of proteins with histidine tag is the most commonly used method because of its relatively small size and charge of the polyhistidine affinity tag ensure that protein activity is rarely affected (Terpe, 2003). We are interested in the characterization of phage K11 lysozyme, a bifunctional enzyme homologous with the widely studied T7 lysozyme. In this study, we described the construction, expression and purification of hexahistidine-tagged phage K11 lysozyme. Our results indicated that the purification tag did not affect the enzymatic activities of K11 lysozyme. The amidase activity of the tagged K11 lysozyme is approximately $55 \%$ greater than the previously purified non-tagged lysozyme (Junn et al., 2005). This result might be attributed in the presence of the tag that could be helpful in stabilizing the enzyme. Previous purification of K11 lysozyme in our laboratory resulted in extensive aggregation and this might affect amidase activity. On the other hand, its ability to inhibit transcription by RNAP in vitro is almost identical with the non-tagged lysozyme. Previously, a hexahistidine-tagged T7 lysozyme was purified and characterized in our laboratory and results also revealed that tagged T7 lysozyme possessed activities that are almost indistinguishable with the non-tagged enzyme. Considering these results, we assumed that utilization of such purification tag would likewise be tolerated in the construction of chimeric enzymes between the two phage lysozymes. Thus, we exploited this affinity in expressing and purifying the chimeric constructs.

The lysozymes encoded by phage T7 and K11 show a high degree of homology $(75 \%)$. Both are bifunctional enzymes that exhibit amidase activity and transcriptional inhibitory activity which are not separated into independent domains. The amidase activities of $\mathrm{T} 7$ and K11 lysozymes are comparable using E. coli as substrate. However, their ability to inhibit transcription in vitro is specific with their respective RNAPs. Another difference between T7 lysozyme and K11 lysozyme is their solubility upon expression in E. coli. T7 lysozyme is mostly found on soluble fractions but K11 lysozyme aggregates as inclusion bodies. The insolubility of K11 lysozyme in E. coli may have been caused by its difference in amino acid sequence and in natural host (Klebsiella for the phage K11) as the case of k11 RNAP (Han et al., 1999)

Domain swapping between T7 and K11 lysozymes generated two chimeras that were both efficiently overexpressed in $E$. coli and enzymatically active. However, both chimeras were mostly expressed as inclusion bodies. The T7K11-lysozyme chimera exhibited amidase activity that is almost identical with the parental enzymes while the K11T7-lysozyme showed an increased activity that is approximately $45 \%$ greater than parental enzymes. The increased amidase activity of K11T7lysozyme could be due to a more favorable structure adapted by the chimera that allows a more efficient binding of the amidase substrate. Furthermore, although both chimeras have the same optimum $\mathrm{pH}$ as the wild-type enzymes, they showed a significant variation in thermal stabilities from their parent enzymes. At $75^{\circ} \mathrm{C}$, the residual amidase activity of T7K11lysozyme and K11T7-lysozyme is $63 \%$ and $53 \%$, respectively but the parental enzymes are completely inactivated at this temperature. Inactivation of the chimeras was observed at $85^{\circ} \mathrm{C}$. Generally, hydrophobic interaction inside a protein molecule is an important factor in stabilizing its structure. Heat stability may be influenced by only a few amino acids substitutions (Singh and Hayashi, 1995). The thermal stability of the chimeras might be possibly due to a significant increase in their hydrophobic properties. Hence, prediction of their structures could provide more insight on their improved properties.

It was previously reported that the $\mathrm{N}$-terminus of $\mathrm{T} 7$ lysozyme is the site of the interaction with T7 RNAP and deletion mutant lacking amino acid residues 2-5 was found to be completely deficient in binding and inhibiting transcription while retaining full amidase activity (Cheng et al., 1993). The chimeras showed transcription inhibition activities that are similar to the parent lysozymes depending on the source of their N-terminal. The T7K11-lysozyme containing a T7 Nterminal inhibits transcription that is comparable with $\mathrm{T} 7$ lysozyme. On the other hand, K11T7-lysozyme, which contains a K11 N-terminal showed the same magnitude of transcription inhibition as K11 lysozyme. The inhibitory effect exhibited by the chimeras implies that the N-terminal of the parental lysozymes is responsible for transcription inhibition specificity. Since it was already established that the Nterminal of T7 lysozyme is the site of binding with T7 RNAP, these results confirmed our previous assumption that the $\mathrm{N}$ terminal of K11 lysozyme is also the binding site of K11 RNAP.

In summary, we were able to construct vectors that efficiently express hexahistidine tagged phage K11 lysozyme and chimeric lysozymes. We were also able to develop a simple method in purifying these enzymes directly from the crude cell lysates using $\mathrm{Ni}^{2+}$ affinity chromatography. Domain swapping technique demonstrated that the N-terminals of T7 and K11 lysozymes determine their specificity with respect to their ability to inhibit transcription by phage RNAP in vitro. 
On the other hand, their amidase activity is not localized on the swapped region and amidase binding could be somewhat dependent on critical residues that are located randomly within the enzyme. These residues are identified in T7 lysozyme and conserved in K11 lysozyme. Site-directed mutations on K11 lysozyme at these sites, therefore, could provide a better understanding on its structure and amidase activity relationship.

Acknowledgment This work was supported by grants from Ministry of Commerce, Industry and Energy of Republic of Korea through the Bio-Medicinal Resource Research Center at Pai Chai University.

\section{References}

Berger, N., Heller, A. E., Stormann, K. D. and Pfaff, E. (2001) Characterization of chimeric enzymes between caprine arthritisencephaltis virus, maedi-visna virus and human immunodeficiency virus type 1 intergrases expressed in E. coli. J. Gen. Virol. 82, 139-148.

Brown, J. E., Klement, J. F. and MacAllister, W. T. (1986) Sequences of three promoters for the bacteriophage SP6 RNA polymerase. Nucleic Acids Res. 14, 3521-3526.

Chamberlin, M., McGrath, J. and Waskell, L. (1970) New RNA polymerase from Escherichia coli infected with baceriophage T7. Nature 228, 227-231.

Cheng, X., Zhang, X., Pflugrath, J. W. and Studier, F. W. (1993) The structure of bacteriophage T7 lysozyme, a zinc amidase and an inhibitor of T7 RNA polymerase. Proc. Natl. Acad. Sci. 91, 4034-4038.

Clark, E. D. (1998) Refolding of recombinant proteins. Curr. Opinion Biotech. 9, 157-163.

Dietz, A., Weisser, H.-J., Kössel, H. and Hausmann, R. (1990) The gene for Klebsiella bacteriophage K11 RNA polymerase: Sequence and comparison with the homologous genes of phages T7, T3, and SP6. Mol. Gen. Genet. 221, 283-286.

Dunn, J. J. and Studier, F. W. (1983) Complete nucleotide sequence of bacteriophage T7 DNA and locations of T7 genetic elements. J. Mol. Biol. 166, 477-535.

Han, K. G., Lee, S. S. and Kang, C. (1999) Soluble expression of cloned phage K11 RNA polymerase gene in Escherichia coli at a low temperature. Protein Express. Purif. 16, 103-108.

Han, K. G., Kim, D. H., Junn, E., Lee, S. S. and Kang, C. (2002) Identification of bacteriophage K11 genomic promoters for K11 RNA polymerase. J. Biochem. Mol. Biol. 35, 637-641.

Huang, J., Villemain, J., Padilla, R. and Sousa, R. (1999) Mechanisms by which T7 lysozyme specifically regulates T7 RNA polymerase during different phases of transcription. $J$. Mol. Biol. 293, 457-475.

Ikeda, R. A. and Bailey, P. A. (1992) Inhibition of T7 RNA polymerase by $\mathrm{T} 7$ lysozyme in vitro. J. Biol. Chem. 267, 20153-20158.

Inouye, M., Arnheim, N. and Sternglanz, R. (1973) Bacteriophage T7 lysozyme is an N-acetylmuramyl-L-alanine amidase. $J$. Biol. Chem. 25, 7247-7252.

Jeruzalmi, D. and Steitz, T. A. (1998) Structure of T7 RNA polymerase complexed to the transcriptional inhibitor $\mathrm{T} 7$ lysozyme. $E M B O$ 17, 4101-4113.

Junn, H. J., Youn, J., Suh, K. H. and Lee, S. S. (2005) Cloning and expression of Klebsiella phage K11 lysozyme gene. Protein Express. Purif. 42, 78-84.

Moffatt, B. A. and Studier, F. W. (1987) T7 lysozyme inhibits transcription by T7 RNA polymerase. Cell 49, 221-227.

Rosa, M. D. and Andrews, N. C. (1981) Phage T3 DNA contains an extra copy of 23 base-pair phage T7 RNA polymerase promoter sequence. J. Mol. Biol. 147, 41-53.

Singh, A. and Hayashi, K. (1995) Construction of chimeric $\beta$ glucosidases with improved enzymatic properties. J. Biol. Chem. 270, 21928-21933.

Terpe, K. (2003) Overview of tag protein fusions: from molecules and biochemical fundamentals to commercial systems. Appli. Microbiol. Biotechnol. 60, 523-533.

Zhang, X. and Studier, F. W. (1995) Isolation of transcriptionally active mutants of T7 RNA polymerase that do not support phage growth. J. Mol. Biol. 250, 156-168.

Zhang, X. and Studier, F. W. (2004) Multiple roles of T7 RNA polymerase and $\mathrm{T} 7$ lysozyme during bacteriophage $\mathrm{T} 7$ infection. J. Mol. Biol. 340, 707-730. 